\title{
Explore Novel Renewable Energy Generation via Applications of Thermoelectric Technology
}

\author{
A. J. Jin ${ }^{1,2} \&$ Y. M. Zhang ${ }^{2}$ \\ ${ }^{1}$ Solar Thermal Energy Division, China Huaneng Clean Energy Research Institute, Beijing, China \\ ${ }^{2}$ Ningbo Inst. of Industrial Technology, Acad. of Chinese Science, Zhejiang Province, China \\ Correspondence: A. J. Jin, Solar Thermal Energy Division, China Huaneng Clean Energy Research Institute, \\ Huaneng Innovation Base Laboratory Bldg-A FutureTechCity, Changping, Beijing 102209, China. Tel: \\ 86-186-0069-9878. E-mail: ajjin@hnceri.com
}

Received: April 12, 2017

Accepted: April 26, 2017

Online Published: May 31, 2017

doi:10.5539/enrr.v7n2p131

URL: https://doi.org/10.5539/enrr.v7n2p131

\begin{abstract}
This paper presents systematic studies of the renewabale energy [RE] technologies and focuses on thermoelectric (TE) technology. The invention of a new equipment is applied to thermoelectric modules (TEM) and demonstrates important capabilities such as in-situ characterization of I-V curve, P-V curves, and TE efficiency. Many variales are invetsigated in order to maximize the output of TE power. Experimental data show that the TE power output is scalable/ additive for multiple TE systems. Multi-stack structures are invetsigated in order to improve the TE efficiency. One of the hightlights of this paper is to generate a large output of TEPG system up to $1 \mathrm{~kW}$ power. The TE technology is one of the most important RE technologies that has advantages of being renewable, possessing low-noise/ no-moving parts among other features, and having versatile and special applications with TEM. Several sets of the thermoelectric power generation (TEPG) systems are developed with various TEMs for many different applications. Finally, the TEPG is characterized for the experimental investigation and data analysis, followed by summaries and conclusion based on the data.
\end{abstract}

Keywords: renewable, energy harvesting, thermoelectric, multi stack, clean tech

\section{Introduction}

The order to combat or control the climate change in part, the international efforts have recently achieved enough signatures from nations that is known as the Paris Agreement in 2016. Extensive the research and development has been dedicated to renewable energy technologies (Brass, Carley, MacLean, \& Baldwin, 2012; Clark III, 2010) by scientists and engineers. Intense studies are conducted both theoretically and experimentally (Chen, 2011; Deng \& Liu, 2009; Tritt \& Subramanian, 2006) on the promising solid-state solar thermal electric generation. Moreover, there are extensive researches recently dedicated to study the thermoelectric power generation (TEPG) in producing power ranges from small to large. In contrast to the solar technology that has solar panels collect photons energy and converts it into electricity, a very different type of renewable energy (RE) technology is the TEPG that converts heat into electricity. TEPG with thermoelectric modules (TEM) generates energy from renewable energy sources such as solar, geothermal, fire source, and other heat source like waste heat/ energy harvesting. The thermoelectric (TE) energy is advantageous as RE in many ways including in part being noiseless with no moving parts, light weight, no-need or little need for maintenance. The current work endeavors to advance TEPG technology.

To apply a foundation in TEPG, the governing laws of physics is the Seebeck effect (Thomson, 1857). The TE device can be understood by a simplified illustration of the Seebeck effect as follows: it converts thermal energy directly into electrical energy and typically employs a pair of dissimilar TE materials to make a device. The output voltage $\Delta \mathrm{V}$ of a TE device generally increases with the temperature differential $\Delta \mathrm{T}$ linearly as follow. In combination of $\mathrm{N}$-type and P-type materials, $\Delta \mathrm{V} \cong \mathrm{S}_{\mathrm{NP}} * \Delta \mathrm{T}$; where the constant, called the Seebeck coefficient (SNP), is related to the materials properties (pairs).

In addition to the efficiency of TEM and/or TEPG, the output power level is another important factor. The power level can be achieved through integration of a large number of TE devices, the efficiency is determined by temperature differential and by a dimensionless figure of merit ZT. ZT is characterized by several material 
parameters such as the Seebeck coefficient (S), thermal conductivity, and electrical resistivity (Mao, Liu, \& Ren, 2016; Liu et al., 2015; Zhao et al., 2010). The total power output equals to the total heat input multiplied by the efficiency. In the later section(s), authors will show that the properties of most TE semiconductors depend upon temperature, e.g., a single TE material typically operates well in about a couple of hundred degrees of temperature window (Crane, Kossakovski, \& Bell, 2009).

There are numerous applications for TEPG ranging from the bio-thermoelectrics to the deep-space power supplies. For example, TEPG delivers key power supply with radioisotope enabled TE output which delivers $350 \mathrm{~W}$ for the Appolo mission to the Mars. In view of the technology pain, the author has studied the pain-points in industry and has highlighted some challenges in this article. If TEM/TEPG must be economic, one significant factor is that the efficiency should achieve some system level or, e.g., with a figure of merit, ZT $>=2$. For example, an efficient TE product helps keep cost down in general and stay competitive.

Finally, based on the research project of a systematic study, the article will cover the following reports: illustrate the experimental setup, measure data, analyze and understand the implication, discuss results, and draw conclusions.

\section{Systematic Studies}

Systematic studies of the TE system are reported as follows.

\subsection{Experimental Setup}

To start experimental discussion, a thermoelectric module is a circuit module that contains a large number of thermoelectric devices together to generate a significant amount of output power. The setup of an operating TE generator includes multiple levels of a TE system hierarchy as follows. A TE device is typically composed of two dissimilar TE legs as illustrated in Figure 1a). The temperature differential causes a net current in the P- and $\mathrm{N}$ - type TE legs. A typical TEM integrates a large number of TE devices shown in Figure 1b). The heat flows through the temperature difference across the device. As devices are connected either in serial, in parallel, or in combination circuit per design, the thermal energy generates a total electromotive force across the two electrical lead as shown. Figure 1a) and Figure 1b) illustrates the principles of TE device operations.

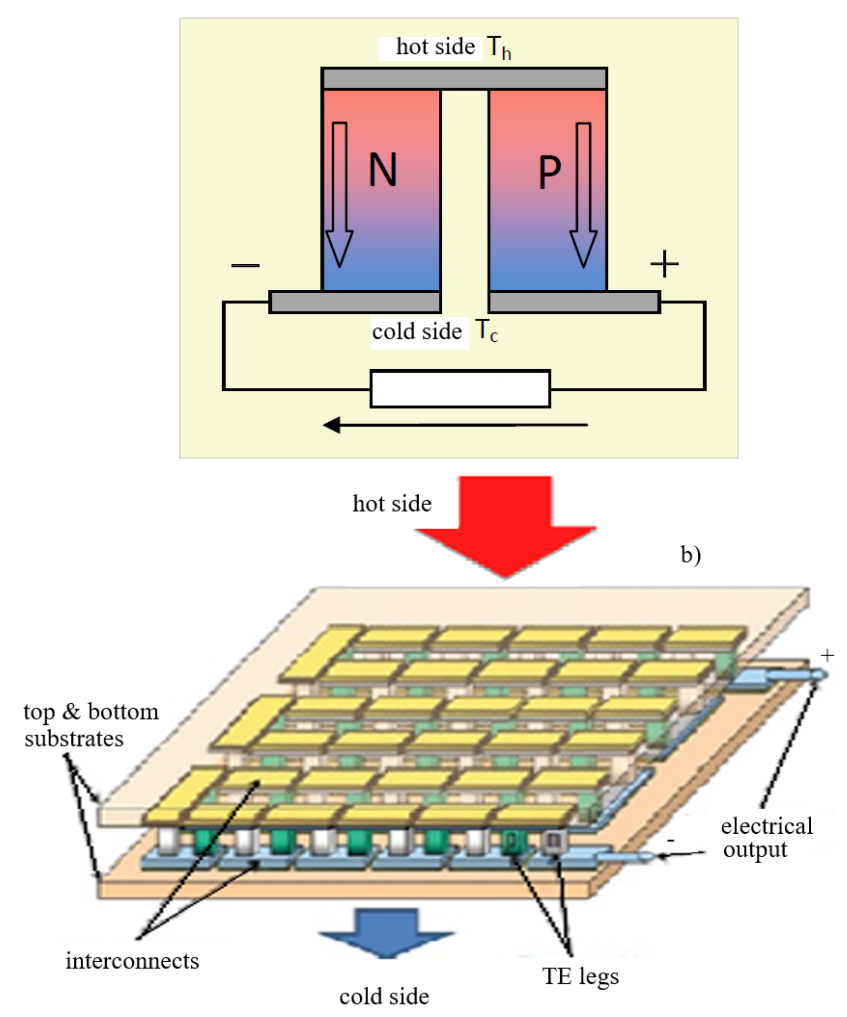

Figure 1. 1a) a schematic illustrates a basic TE device where it p- and n-type TE elements are set up a temperature differential; where the electric power is generated. 1b) TEM is an integration of TE devices connected to provide increased power output 
Variation of the TE system depends on technology applications; e.g., some interesting illustration may refer to Jin et al. (2013). When TEPG is built, researchers have applied careful thermal management methods and built systems at various power levels. The methods include the fine insulation mentioned to be shown later in this section, and good uniformity conducting heat [for both the cooling surface and heating surface]. The hot side normally employs one of the heating choices of the infrared heat, of fire-heat, of electric heaters, of solar thermal, or of liquefied natural gas (LNG), and other methods. The LNG is normally warmed to use in cooking as well as electricity generation and other uses. The above choices are laid to establish the groundwork of prototypes for various (potential) applications.

The in-situ characterization station for thermoelectric module (ICSTEM) is an instrument to measure in situ the conversion efficiency of TEM. It is comprised of the following tests: measure the output power P, current I, inner resistance $\mathrm{R}$ and the cold-end heat flux $\mathrm{Q}_{\mathrm{cl}}$ of the thermoelectric module under power generation status. The efficiency is defined as the ratio of the electric power output, $\mathrm{P}$, to the heat input, $\mathrm{Q}_{\mathrm{h}}$, in the hot side of the thermal electric device.

The ICSTEM is carefully designed so that the physics of heat flow can be well-described by that the heat flux flows through the $\mathrm{z}$-direction and that the thermoelectric conversion efficiency can be measured conveniently in-situ in real time. Theoretically, the cooling capacity Qc is calculated as follows:

$$
Q_{c}=S_{N P} T_{c} I-\frac{1}{2} I^{2} R-k\left(T_{h}-T_{c}\right)
$$

Where $S_{N P}$ is TEM Seebeck coefficient, $T_{h}$ and $T_{c}$ are its temperatures at hot-side and cold-side, I current, $R$ resistance, $\mathrm{k}$ thermal conductance. The cooling capacity is maximal when a TEM has the same cold-side and hot-side temperatures.

When the ICSTEM is designed (Mao, Liu, \& Ren, 2016), TEM should be placed flatly and be parallel with the two relative edges of the heat flux sensors near its cold side. Referring to Figure 2, the flat surface of the cold plate can be vertically adjusted by the four set-screws independently; each set screw has a fine threaded resolution of ten microns and below. The torque pressure can be set on the flat surface via the torque wrench as shown which could guarantee the peak level TE performance at given pressure. Figure 3 illustrates a typical four-points probe that measures efficiency of TE device/ legs in open-air condition. A temperature differential is applied from both of the designated top- and bottom- plates. The actual temperatures on every sides are measured in real time with two thermocouples (TCs). The efficiency is measured as a function of temperature. $\mathrm{I}-\mathrm{V}$ and $\mathrm{P}-\mathrm{V}$ features will be investigated later.

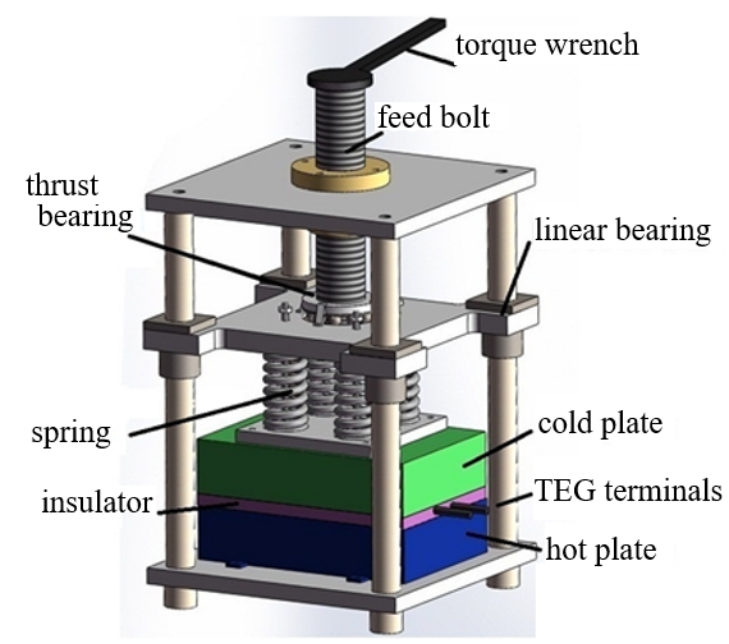

Figure 2. ICSTEM schematic illustrates the TEG sandwiched by cold plate and hot plate where the efficiency is measured in situ 


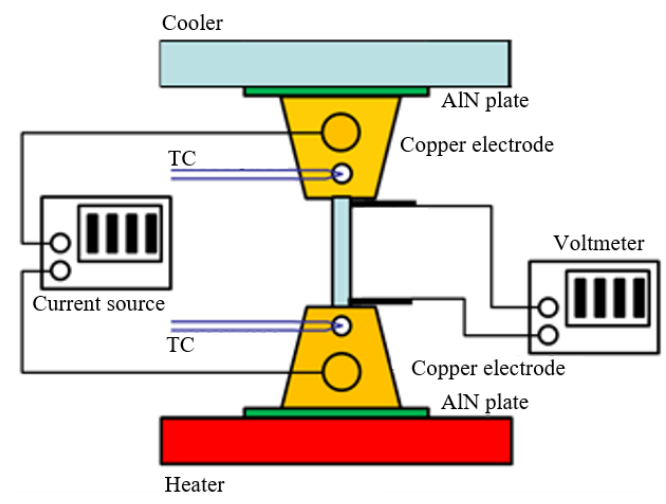

Figure 3. the schematic is a four-points probe to measure I-V curves of TE pairs. The electrical current and the voltage across TE leg is measured by the meters. Copper and/or AIN are employed for the best contact either electrically and/ or thermally

The system has relatively small size, is thermally insulated at peripheral surfaces, and has good uniformity conducting heat for the cold surface and hot surface. The hot side controls the temperature $\mathrm{T}_{\mathrm{h}}$ with specific heat-source; the cold side controls temperature $T_{c}$ of the thermoelectric module under cooling conditions. In this case, the cold side employs one of cooling choices [e.g., water, or liquid nitrogen cooled]. High infrared reflection and insulation materials, for example, may be chosen as the alternating layers of thin metal sheet and thin glass fiber sheet [or insulator layer]. This can significantly reduce the influence of the heat leakage in heat conduction. The in-situ characterization station is carefully designed so that the physics of heat flow can be well described by a 1D heat flow model (Solla \& Riedel, 1981) that the heat flux flows through the z-direction.

\subsection{Analysis and Data Collection}

Many studies are conducted on TEM and TEPG system(s) and characterized in this article. When $\mathrm{T}_{\mathrm{h}}$ reaches 300 deg-C, the output power has reached $300 \mathrm{~W}$ or more per unit; it exceeds $1000 \mathrm{~W}$ with a group of four units. Researchers have designed, first built, and tested in the laboratory as well as tested in the field. The prototype has delivered the output power that is predicted by simulation results. The simulation model has been conducted with SolidWorks ${ }^{\circledR}$ (Note 1) thermal physics software with proprietary algorithm.

A number of heat sources and/or cooling mechanism are employed with various extent of success to demonstrate a range of TEPG power output. The author has investigated many options as follows. For example, the infrared heating is used as a heat source, the direct-fired diesel burner employed, or the LNG burner employed as well.

The output power is plotted in the figure as a function of the hot side temperature. Moreover, a range of heat sources from the automotive tailpipe energy harvesting to solar heater are employed for the investigation.

Figure 4 illustrates the relationship of power output and temperature built and tested with commercial TE modules (Note 2). Based on the combined multiple-unit studies, the total power output is superimposed well in either serial or parallel configuration and typically equals to a sum of all units of individual TEPG (Jin et al., 2013). The empirical analysis based on the data at above generally concurs with results derived the SolidWorks ${ }^{\circledR}$ model.

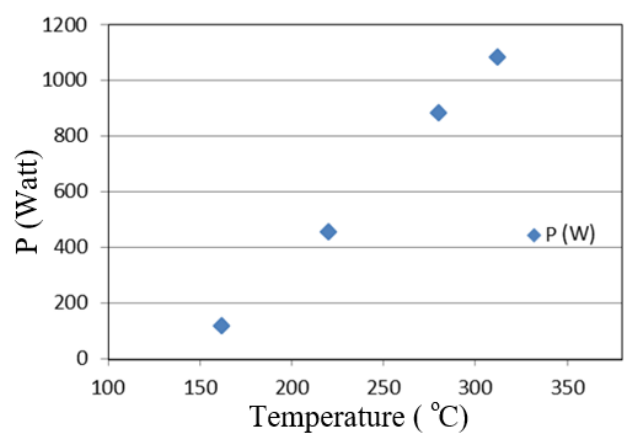

Figure 4. Lab tests for power output of TEPG show that its maximum output depends nearly linearly upon the temperature in the tested range 
The TE efficiency is demonstrated in Figure 5; it shows the relationship of TE efficiency as a function of the hot side temperature. It is expected that the efficiency increases with the temperature. Moreover, many tests for different modules covering various temperature ranges have been demonstrated and overlaid in the same figure. In addition to the nearly linear relationship of the efficiency vs temperature, the TE devices have exhibited TE efficiency from a few percent to ten percent. The TE efficiency of dual stack is approximately ten percent and lays above this chart. The dual stack efficiency lays above the chart at 450 degrees Celsius. Further studies on details of the Efficiency -vs- temperature relationship are in progress.

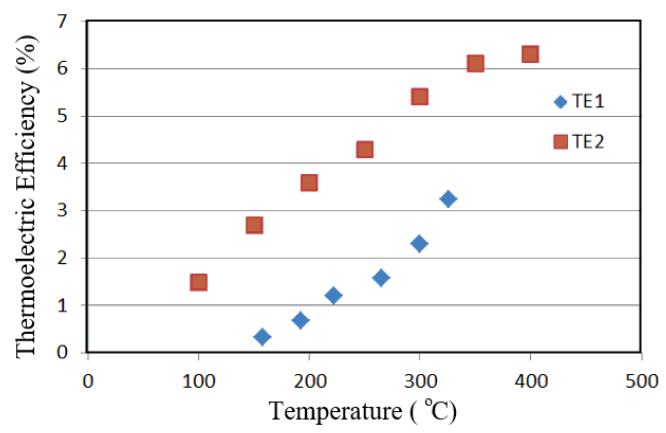

Figure 5. The charts demonstrate the dependence of efficiency versus temperature at their hot side of TE systems.

TE1 and TE2 studies the efficiency of off-the-shelf sample-1 and sample-2, respectively

\subsection{Discussions of TE Stacks}

Experiments \& investigation are undertaken as follows.

Figure 6 illustrates the dependence of efficiency upon the length ratio ( $\mathrm{x}$ ) for both P-type and N-type TE materials, where $x$ is the joint position in the ratio of the length where segmented legs locate at. Figure 6a) shows P-type efficiency as a function of $\mathrm{x}$ at three temperatures settings; Figure $6 \mathrm{~b}$ ) shows N-type as a function of $\mathrm{x}$. The TE efficiency is $11.8 \%$ at $550 \mathrm{deg}-\mathrm{C}, \mathrm{T}_{\mathrm{h}}$, for P-type; and it is $9.7 \%$ at $550 \mathrm{C}$ for N-type.
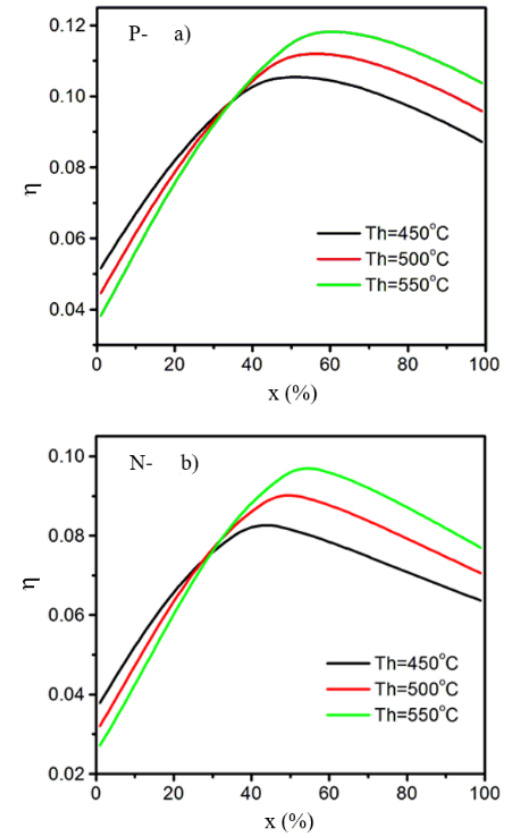

Figure 6. Illustrates efficiency for both P-type and N-type materials. 6a) P-type efficiency is exhibited as a function of $x$ at 3 different temperature settings; 6b) $\mathrm{N}$-type efficiency as a function of $\mathrm{x}$ at 3 -settings
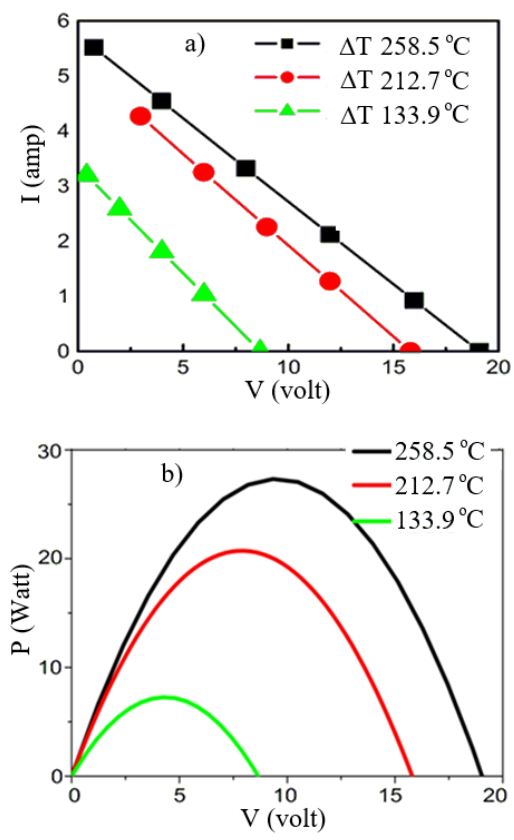

Figure 7. Illustrates electrical features of TE devices at below: 7a) shows I-V curves exhibiting typical traces of a low resistance current supply;.7b) shows P-V curves of typical resistive power traces 
In Figure 7, each TEM device is characterized for its fundamental electrical property traces in terms of current (I) versus voltage $(\mathrm{V})$ curves, i.e., $\mathrm{I}-\mathrm{V}$ curves, and in terms of power output [at maximum value point] $\mathrm{P}$ versus $\mathrm{V}$, i.e., P-V curves. Figures have plotted typical data of a system at three different temperature settings at hot side as follows. The power output increases with the temperature $T_{h}$.

\subsection{Results and Discussion}

The TEM is built in the laboratory. It can produce electricity which is demonstrated at various output specs up to $1 \mathrm{~kW}$ level with wide choices of heat sources.

Researchers have built multi stack with the available TE materials. The computer simulation with the finite elements method of triple stack TEPG predicts that the efficiency can achieve over 19\% (Liu \& Jin, 2015; Liu \& Jin, 2013).

This article shows that the dual stack system is built and has achieved $10 \%$ efficiency in the lab. Conversion of the thermal energy into electricity employs solid-state devices that has no moving parts, and expands use of the energy resources vastly. Similar to other distributed power or RE power plants, TEPG is environmental that is also beneficial and convenient for integration into the smart grid.

In order to achieve improved efficiency and/or power level, the chosen TE materials are optimized suitably for segmented-legs. The TE leg preparation has enabled superior efficiency of the TEM (Ursell \& Snyder, 2002).

As it is shown in the Table I, the dual stack materials are consisted of PbTe based and BiTe based TE materials. The first types are PbTe based TE materials [and its variation stoichiometry] include both in P-type and in N-type being optimized for a medium temperature range; the BiTe TE materials (and its variation stoichiometry) include both in P-type and in N-type optimized for a low temperature range. The dual stack P- and N-type PbTe/ BiTe based segmented legs are joint together at high temperature through hard soldering that otherwise cannot be weld together. The dual stack TE system exhibits the enhancement of figure of merit, ZT of the system as shown in Table I at below. The ZT is tabulated for several TE materials covering high temperature.

Careful studies measure the efficiency of $10 \%$ when a temperature differential has reached 400 degrees. The table employs temperature unit kelvin $(\mathrm{K})$, where $\mathrm{n} / \mathrm{K}=\mathrm{m} / \mathrm{C}+273.15$.

Table I. Figure of merit ZT of TE materials/ legs is tabulated at below: (P_PbTe: $\mathrm{Pb}_{0.94} \mathrm{Sr}_{0.04} \mathrm{Na}_{0.02} \mathrm{Te}$, N_PbTe: $\mathrm{Pb}_{0.94} \mathrm{Ag}_{0.01} \mathrm{La}_{0.05} \mathrm{Te}$, P_BiTe: $\mathrm{Bi}_{0.5} \mathrm{Sb}_{1.5} \mathrm{Te}_{3}$, N_BiTe: $\left.\mathrm{Bi}_{2} \mathrm{Te}_{3}\right)$

\begin{tabular}{|c|c|c|c|c|c|c|c|}
\hline \multicolumn{2}{|c|}{ P- $\mathrm{PbTe}$} & \multicolumn{2}{|c|}{$\mathrm{N}-\mathrm{PbTe}$} & \multicolumn{2}{|c|}{ P- $\mathrm{BiTe}$} & \multicolumn{2}{|c|}{$\mathrm{N}-\mathrm{BiTe}$} \\
\hline $\mathrm{T}(\mathrm{K})$ & $Z T$ & $\mathrm{~T}(\mathrm{~K})$ & $Z T$ & $\mathrm{~T}(\mathrm{~K})$ & $Z T$ & $\mathrm{~T}(\mathrm{~K})$ & $Z T$ \\
\hline 321 & 0.17 & 320 & 0.18 & 327 & 0.88 & 326 & 0.83 \\
\hline 422 & 0.48 & 420 & 0.39 & 379 & 0.95 & 373 & 0.94 \\
\hline 522 & 1.13 & 524 & 0.71 & 431 & 0.91 & 423 & 1.11 \\
\hline 622 & 1.76 & 620 & 1.09 & 482 & 0.72 & 524 & 0.92 \\
\hline 722 & 1.86 & 726 & 1.44 & & & & \\
\hline 822 & 2.12 & 824 & 1.51 & & & & \\
\hline 922 & 2.05 & 924 & 1.41 & & & & \\
\hline
\end{tabular}

If the unit cost continues to drop from the current price tag of the off-the-shelf TEM, and if ZT 2.0, TEPG can possibly achieve advantage competitively in a scale of economy. There are extensive studies to improve ZT with new TE materials in the literature (Mao, Liu, \& Ren, 2016; Mao, Liu, \& Ren, 2016). It is the author's belief that the current TEPG technology is still in the early stage with a limit in TE efficiency attributes of the available materials.

ZT is related to material properties including electrical conductivity, thermal conductivity, and Seebeck coefficient. Refer to Table I, the favorable working temperature of PbTe based materials is from $573 \mathrm{~K}$ to $873 \mathrm{~K}$. The dimensionless figure of merit in the relevant temperature range $(\mathrm{ZT}=\mathrm{S} 2 / \rho \kappa)$ is about 1.5 2.1 for P-type and 0.9 1.4 for N-type. The BiTe based TE materials cover temperature range lower than $\mathrm{PbTe}$. The dimensionless figure of merit in the relevant temperature range is about $0.7 \sim 0.9$ for P-type and $0.8 \sim 1.1$ for $\mathrm{N}$-type. The upshot of experimental data is that the multi stack of TEM improves the system TE efficiency.

Author has achieved hundreds of watts in power output for each TEPG system unit, and has produced $1 \mathrm{~kW}$ by additively connecting a few units together. Moreover, by utilizing the currently available thermoelectric materials and employing dual stack TEMs, the above data demonstrate that the dual stack TEM leads to a higher efficiency than a single stack as expected. 
Combined results of multi stack TE materials have utilized P_PbTe, N_PbTe, P_BiTe, and N_BiTe. The discovery includes dual stack TE device of $10 \%$ efficiency; computer modeling delivers $19 \%$ efficiency for triple stack TEM.

Finally, among many applications, author has investigated many important parameters/ conditions as follows: operating pressure setting, in situ efficiency study, and the materials research and TEPG scalability (Jin et al., 2013). More work to improve both the efficiency and production cost is in progress.

\subsection{Scalability and Additivity}

The additivity of TEG systems and its trends are investigated for TEG systems that work out generally very well. For example, in one case the temperature differential is set at about 95 degrees-C for two of the TEG prototypes/ systems. After taken standard measurements, data show as follows: prototype-A delivers 19.95 Watt; prototype-B delivers 19.45 Watt. The data also shows that the total power satisfies the law of additivity. The total power in serial connection of the two systems yields an output of $39.27 \mathrm{~W}$ and the power in parallel connection of the two systems yields an output of $39.11 \mathrm{~W}$. Likewise, the case at 62 degrees-C satisfies the additivity, too.

The errors are shown to be insignificant; multiple tests and lead us to conclude with a level of confidence over $99.5 \%$ that the superposition meets the law of additivity. The error bar for the power data is very small.

The table II at below illustrates the experimental proof of the additivity law. The additivity is measured/ repeated for several of the tests where each and every one works well for them. In other words, this is a typical behavior shown in the table II.

Table II. Demonstration of power scalability in order to superimpose TEG prototype/ systems

\begin{tabular}{|c|c|c|c|c|}
\hline$\Delta \mathrm{T}(\mathrm{deg})$ & $\mathrm{PA}(\mathrm{W})$ & $\mathrm{PB}(\mathrm{W})$ & Serial(W) & Parallel(W) \\
\hline $62 \mathrm{C}$ & 8.98 & 8.22 & 17 & 17.07 \\
\hline $.95 \mathrm{C}$ & 19.95 & 19.45 & 39.27 & 39.11 \\
\hline
\end{tabular}

\subsection{Clamping Force}

Over a wide range of the force applied, the output power could be very significant. A typical TE module demonstrates the force response curve, which is shown in Figure 8, as follows.

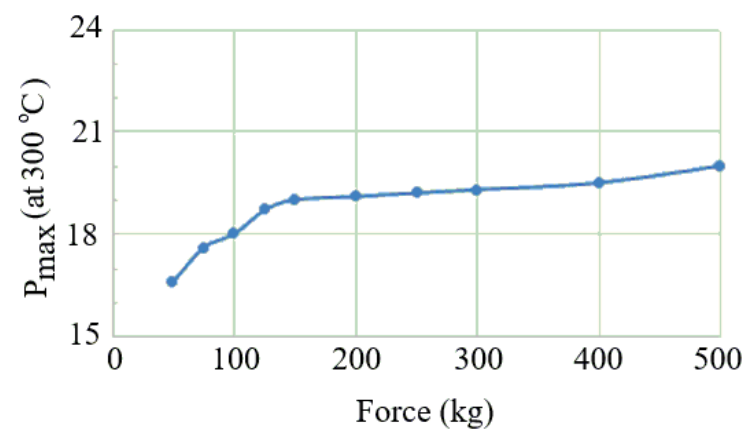

Figure 8. Maximum output power curve for a module under different clamping forces, with a cold side of $25^{\circ} \mathrm{C}$

Referring to the chart, the experimental curve is taken, where the hot side temperature is $300 \mathrm{C}$ and the cold side temperature is $25 \mathrm{C}$.

Researchers discover a typical relationship of TEM output power and the applied clamping force. Near the low force side, the power increases significantly with the force. The curve exhibits a total variation of twenty percent that is also observed for many times at various temperature setting.

As it is shown in the chart, the force chosen at $250 \mathrm{~kg}$ works well where the curve essentially flattens. Moreover, the clamping force is set below $350 \mathrm{~kg}$ in order to limit any risks of failure caused by crack or other defects at very high clamping forces. At a wide temperature window, the $250 \mathrm{~kg}$ force is a good setting choice for the force which equals to $8.0 \mathrm{~kg} / \mathrm{cm}^{\wedge} 2$ of the experimental setting of pressure. 
The causes for the chart trends has been believed to do with the defect or factors of contact interface behavior such as variation in electrical contact resistance, air gap, interfacial structures, and changes caused by packaging materials.

The afore-mentioned defect or imperfection [of TEM] could be optimized through proper setting of the clamping force; and the optimization should reduce extra parasitic losses with optimized setting with low parasitic losses: e.g., low contact resistance, small radiation effects, little inter-diffusion at any junction.

\section{Summaries and Conclusions}

Authors endeavor systematic approaches in the research. They pursue the scientific and technological methodology in building the thermoelectric generation systems.

Many prototypes are built in a wide application range; the output power specs cover over four orders of magnitude up to $1000 \mathrm{~W}$. System optimization enables the authors to successfully build many prototypes. They have studied many features of electrical performance of TEPG system.

An advanced platform of in situ measurements is constructed to study the thermoelectric efficiency. Important technologies based on the systematic research are investigated.

Because the materials are often suitable for a limited T-window [about 200 degrees], a combination of multi-stack TE materials is studied in order to improve the efficiency. TEM can be made with multi-stack approach that provides superior efficiency of the system. For example, studies show that dual stack PbTe class and BiTe class can achieve 10\% efficiency and that, in triple-stacks, TE device can achieve $19 \%$ efficiency. The system scalability and the force factor are identified for parametric settings.

Based on all of the evidences existed in the niche market, the TEPG technology is believed to be on the verge of main stream apps. The high-quality heat helps to maintain the large temperature differential in order to deliver good outputs.

\section{Acknowledgment}

The authors are very appreciative of the support and discussions from Drs. G. Yin, J. Chien, X. SU, and Prof. T. Deng. They gratefully acknowledge contributions of Drs. W. Peng, D. Liu, and D. Lv for the work on an original version.

\section{References}

Brass, J. N., Carley, S., MacLean, L. M., \& Baldwin, E. (2012). Power for development: a review of distributed generation projects in the developing world. Annual Review of Environment and Resources, 37, 107-136. $\mathrm{http} / / / \mathrm{dx}$. doi.org/10.1146/annurev-environ-051112-111930.

Chen, G. (2011). Theoretical efficiency of solar thermoelectric energy generators. Journal of Applied Physics, 109(10), 104908. http://dx.doi.org/10.1063/1.3583182.

Clark III, W. W. (2010). Sustainable communities design handbook: green engineering, architecture, and technology. Elsevier.

Crane, D. T., Kossakovski, D., \& Bell, L. E. (2009). Modeling the building blocks of a $10 \%$ efficient segmented thermoelectric power generator. Journal of electronic materials, 38(7), 1382-1386.

Deng, Y. G., \& Liu, J. (2009). Recent advances in direct solar thermal power generation. Journal of Renewable and Sustainable Energy, 1(5), 052701. http://dx.doi.org/10.1063/1.3212675.

Jin, A. J., \& Peng, W. (2013). Internal reports, CERI/TW-RA-006-13 (2013) of China Hua-Neng Group. Authors has benefited by numerous discussions with partners, peers, and vendors. They appreciate their supports to meet some aggressive project spec. Some of the VLSI processes provided by J. Chien for thick oxide growth, by G. Yin for patterned etch of thick oxide in AMEC Ltd., and by Prof. T. Deng for the filling of both p-type and n-type BiTe.

Jin, A. J., Peng, W., Jin, Y., Liu, D., \& Li, Q. (2013). Research, Development, and Applications of the High-Power Thermoelectric Generation Technology. ARPN Journal of Science and Technology, 3, 901.

Khan, S., Lorenzelli, L., \& Dahiya, R. S. (2015). Technologies for printing sensors and electronics over large flexible substrates: a review. IEEE Sensors Journal, 15(6), 3164-3185.

Li, S., Pei, J., Liu, D., Bao, L., Li, J. F., Wu, H., \& Li, L. (2016). Fabrication and characterization of thermoelectric power generators with segmented legs synthesized by one-step spark plasma sintering. Energy, 113, 35-43. 
Liu, D., \& Jin, A. J. (2013). The computer simulation with the finite elements method of triple stack TEPG predicts that the efficiency can achieve over 19\%". HNCERI internal report.

Liu, D., \& Jin, A. J. (2015). Multi-stack thermoelectric segmentation and optimization: a computation software. SW copyrights Reg. ID.: 2015SR094544 V1.0, China.

Liu, D., Li, Q., Peng, W., Zhu, L., Gao, H., Meng, Q., \& Jin, A. J. (2015). Developing instrumentation to characterize thermoelectric generator modules. Review of Scientific Instruments, 86(3), 034703.

Mao, J., Liu, Z., \& Ren, Z. (2016). Size effect in thermoelectric materials. npj Quantum Materials, 1, 16028. Retrieved from http://www.nature.com/articles/npjquantmats 201628

Mills, J. C., \& Dahlberg, R. C. (1991, January). Thermionic systems for DOD missions. In AIP Conference Proceedings (Vol. 217, No. 3, pp. 1088-1092). AIP. http://dx.doi.org/10.1007/BF01123508

Nolas, G. S., Sharp, J., \& Goldsmid, J. (2013). Thermoelectrics: basic principles and new materials developments (Vol. 45). Springer Science \& Business Media.

Solanki, P. M., Deshmukh, D. S., \& Talele, D. C. (2015). A Critical Review on Operating Variables of Solar Thermoelectric Power Generator. Pratibha: International Journal of Science, Spirituality, Business and Technology (IJSSBT), 3(2), 49-54.

Solla, S. A., \& Riedel, E. K. (1981). Vortex excitations and specific heat of the planar model in two dimensions. Physical Review B, 23(11), 6008.

Thomson, W. (1857). 4. On a Mechanical Theory of Thermo-Electric Currents. Proceedings of the Royal society of Edinburgh, 3, 91-98.

Tritt, T. M., \& Subramanian, M. A. (2006). Thermoelectric materials, phenomena, and applications: a bird's eye view. MRS bulletin, 31(03), 188-198. http://dx.doi.org/10.1557/mrs2006.44.

Ursell, T. S., \& Snyder, G. J. (2002, August). Compatibility of segmented thermoelectric generators. In Thermoelectrics, 2002. Proceedings ICT'02. Twenty-First International Conference on (pp. 412-417). IEEE.

Yin, Z., Huang, Y., Bu, N., Wang, X., \& Xiong, Y. (2010). Inkjet printing for flexible electronics: Materials, processes and equipments. Chinese Science Bulletin, 55(30), 3383-3407.

Zhao, D., Tian, C., Tang, S., Liu, Y., Jiang, L., \& Chen, L. (2010). Fabrication of a CoSb 3-based thermoelectric module. Materials Science in Semiconductor Processing, 13(3), 221-224.

Zhao, L. D., Lo, S. H., Zhang, Y., Sun, H., Tan, G., Uher, C., ... \& Kanatzidis, M. G. (2014). Ultralow thermal conductivity and high thermoelectric figure of merit in SnSe crystals. Nature, 508(7496), 373-377. http://dx.doi.org/10.1038/nature13184.

\section{Notes}

Note 1. SolidWorks is a solid modeling computer-aided design (CAD) and computer-aided engineering (CAE) computer program. SolidWorks Copyright is owned by SOLIDWORKS Corp, USA.

Note 2. Commercial TEM is employed for reference in part, for example, TEM No. TEHP1-12656-0.3 from the manufacturer owned by Thermonamics Electronics (Jiangxi) Corp., Ltd., China.

\section{Copyrights}

Copyright for this article is retained by the author(s), with first publication rights granted to the journal.

This is an open-access article distributed under the terms and conditions of the Creative Commons Attribution license (http://creativecommons.org/licenses/by/4.0/). 